\title{
MicroRNAs 10a and 10b are potent inducers of neuroblastoma cell differentiation through targeting of nuclear receptor corepressor 2
}

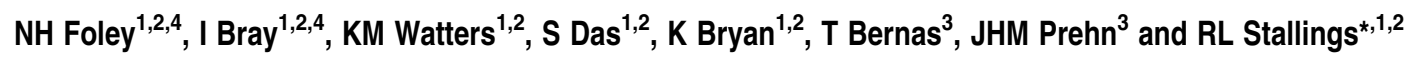

MicroRNAs function as negative regulators of posttranscriptional gene expression, having major roles in cellular differentiation. Several neuroblastoma cell lines can be induced to undergo differentiation by all-trans-retinoic acid (ATRA) and are used for modeling signaling pathways involved in this process. To identify miRNAs contributing to differentiation, we profiled 364 loci following ATRA treatment of neuroblastoma cell lines and found miR-10a and miR-10b to be highly overexpressed in SK-N-BE, LAN5 and SHSY-5Y. Ectopic overexpression of these miRNAs led to a major reprogramming of the transcriptome and a differentiated phenotype that was similar to that induced by ATRA in each of these cell lines. One of the predicted downregulated $\mathrm{miR}-10 \mathrm{a} / \mathrm{b}$ targets was nuclear receptor corepressor 2 (NCOR2), a corepressor of gene transcription, which is known to suppress neurite outgrowth. NCOR2 was experimentally validated as a direct target of miR-10a/b, and siRNA-mediated inhibition of this mRNA alone resulted in neural cell differentiation. Moreover, induction of differentiation could be blocked by ectopic upregulation of NCOR2 using an expression construct lacking the miR-10a/b $3^{\prime}$ untranslated region target site. We conclude that miR-10a/b has major roles in the process of neural cell differentiation through direct targeting of NCOR2, which in turn induces a cascade of primary and secondary transcriptional alterations, including the downregulation of MYCN.

Cell Death and Differentiation (2011) 18, 1089-1098; doi:10.1038/cdd.2010.172; published online 7 January 2011

MicroRNAs are small non-coding RNAs (18-25 nucleotides in length) that act as complex negative regulators of posttranscriptional gene expression by targeting regions of sequence similarity in mRNA $3^{\prime}$ untranslated regions (UTRs), leading to either degradation or translational inhibition of the mRNA. ${ }^{1-3}$ MiRNAs are expressed in a temporal- and spatial-specific manner in the development of the brain, ${ }^{4,5}$ thus contributing to the process of determining neuronal cell identity, as reviewed by Li et al. ${ }^{6}$

Neuroblastomas are pediatric tumors originating from precursor cells of the sympathetic nervous system, which have highly diverse clinical outcomes, ranging from spontaneous regression to rapid progression. Cell lines derived from these tumors, such as SK-N-BE, SHSY-5Y and LAN-5, can be induced to undergo neural cell differentiation by treatment with all-trans-retinoic acid (ATRA) and are often used as a model system to study biochemical pathways involved in the process of differentiation. ${ }^{7}$ ATRA treatment of these cell lines leads to the outgrowth of neurites, the upregulation of markers of neural differentiation such as TUBB3 and GAP43, and a decrease in the rate of cell proliferation. These morphological and functional changes are reflective of the large numbers of protein-coding genes ${ }^{8}$ and miRNAs $^{9}$ that have been altered in cells induced to undergo differentiation by ATRA. Most notably, ATRA leads to immediate transcriptional downregulation of the $M Y C N$ transcription factor before the onset of cellular morphological differentiation, ${ }^{10}$ which further contributes to the remodeling of the transcriptome. MYCN is amplified or expressed at high levels in the cell lines studied, and it is notable that MYCN amplification is one of the most potent genetic predictors of poor clinical outcome for neuroblastoma patients. ${ }^{11,12}$

Recently, it has been demonstrated that some of the miRNAs that undergo expressional changes following ATRA treatment appear to functionally contribute to the differentiation phenotype of neuroblastoma. Laneve et al. ${ }^{13}$ demonstrated that ATRA induced upregulated miRNAs 9, 125a and $125 \mathrm{~b}$ decrease cell proliferation rates through the common targeting of a truncated isoform of the neurotrophin receptor tropomyosin-related kinase C mRNA. It was further shown that miR-125b ectopic overexpression induces neurite outgrowth and neuronal gene expression through the inhibition of multiple mRNA targets. ${ }^{14}$ Another miRNA, which is upregulated in ATRA-treated neuroblastoma cell lines, miR-128, was shown to reduce neuroblastoma cell motility and invasiveness through inhibiting the Reelin and $D C X$ genes. $^{15}$ The miR-17-5p polycistronic cluster has been shown to be downregulated in response to ATRA, ${ }^{16}$ and knockdown of two family members, miR-18a and -19a, results in neuroblastoma cell differentiation through the targeting of

\footnotetext{
'Department of Cancer Genetics, Royal College of Surgeons in Ireland, York House, Dublin, Ireland; ${ }^{2}$ Children's Research Centre, Our Lady's Children's Hospital, Crumlin, Dublin, Ireland and ${ }^{3}$ Department of Physiology and Medical Physics, Royal College of Surgeons in Ireland, York House, Dublin, Ireland

${ }^{*}$ Corresponding author: R Stallings, Department of Cancer Genetics, Royal College of Surgeons in Ireland, York House, York Street, Dublin 2, NA 2, Ireland.

Tel: + 3531402 8533; E-mail: rstallings@ rcsi.ie

${ }^{4}$ Designated as co-first authors.

Keywords: ATRA; MYCN; neuroblastoma; miR-10a; miR-10b; NCOR2

Abbreviations: ATRA, all-trans-retinoic acid; NCOR2, nuclear receptor co-repressor 2; MYCN, v-myc myelocytomatosis viral related oncogene; HOX, homeobox gene; TUBB3, tubulin, beta 3; GAP43, growth associated protein 43; miRNA, microRNA; miR, microRNA; UTR, untranslated regions; GO, gene ontology

Received 14.6.10; revised 05.11.10; accepted 26.11.10; Edited by RA Knight; published online 07.1.11
} 
estrogen receptor- $\alpha .{ }^{17}$ Chen et al. ${ }^{18}$ showed that ATRA induced miR-214 overexpression in neuroblastoma SHSY$5 Y$ cells can modulate neuronal differentiation, and also showed that ATRA repression of miR-7 also induces neurite outgrowth. In addition, it was recently demonstrated that some miRNAs upregulated in response to ATRA target DNA methytransferases, resulting in demethylation and upregulation of specific genes, which are known to be involved with ATRA-induced differentiation. ${ }^{19}$ From all of these studies it is apparent that the contribution of miRNAs towards an ATRAinduced differentiated phenotype is an essential, highly complex, but poorly understood process.

Upregulation of miR-10a in response to ATRA was reported by Beveridge et al. ${ }^{16}$ for SHSY-5Y cells, although this group did not carry out functional studies to assess the role of miR10 family members in ATRA-induced differentiation. Here, we have carried out a detailed functional analysis of the biological effects of miR-10a and $-10 b$, which we determine to be also upregulated in SK-N-BE and LAN-5 cells in response to ATRA. Interestingly, both miR-10a and $-10 \mathrm{~b}$ are imbedded in HOX gene clusters on chromosomes $17 q$ and $2 q$, respectively. $H O X$ genes are transcription factors that are expressed in embryogenesis in distinct regions during head-tail body axis of animal embryos. This cluster of genes is highly important in development, as precise regulation is necessary for efficient differentiation to occur. ${ }^{20}$ In addition to being colocalized within HOX gene clusters, these miRNAs are also known to directly inhibit some of the HOX genes, with

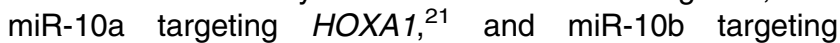
HOXD10. ${ }^{22}$ Tsang et al. ${ }^{23}$ have previously shown neuronalenriched miRNAs tend to be coexpressed with their target genes, suggesting a role for these miRNAs in neuronal homeostasis. Here, we investigate the importance of these miRNAs in ATRA-induced neuroblastoma cell differentiation, including the identification of a direct target gene, nuclear receptor corepressor 2 (NCOR2; also known as SMRT), whose silencing recapitulates an ATRA-induced differentiated phenotype.

\section{Results}

Differential expression of miRNAs after retinoic acidinduced differentiation of SK-N-BE cells. SK-N-BE, SHSY-5Y and LAN-5 neuroblastoma cells treated with ATRA exhibited extensive outgrowth of neurites (Figure 1). Further analysis of SK-N-BE cells revealed that ATRAinduced differentiation resulted in the overexpression of markers of neuronal differentiation (GAP43 and TUBB3), a decrease in MYCN mRNA and protein, and a significant decrease in cell growth, all well-known features of ATRAinduced differentiation (Supplementary Figure 1). In order to gain further insight into miRNAs that might be involved in the process of neural cell differentiation, we profiled 364 mature miRNAs in ATRA-treated and -untreated SK-N-BE cells, using TaqMan low-density arrays (Applied Biosystems, Carlsbad, CA, USA). ATRA-induced differentiation had a major impact on the expression levels of numerous miRNAs, with 30 loci having statistically significant expressional increase $(>1.5$ fold; $P<0.05)$ and 26 loci exhibiting expressional decrease $(>1.5$ fold; $P<0.05)$ in two biological replicates (Supplementary Figure $2 a$ ).

MiRNAs with the highest expressional changes ( $>10$ fold at day 7) included miR-132, miR-10a and miR-10b. Although miR-132 was also highly-upregulated in response to ATRA, Chen et al. ${ }^{18}$ reported that ectopic overexpression of miR-132 in neuroblastoma cells had no consistent effect on neurite outgrowth, so we focused our studies on miR-10a and -10b for which functional studies in neuroblastoma cells have never been reported. Both of these miRNAs were also significantly upregulated in SHSY-5Y and LAN5 cells in response to ATRA (Supplementary Figure $2 b$ and $c$ ), indicating that this is a general feature of neuroblastoma cell lines undergoing ATRAinduced differentiation. The high differential expression of $\mathrm{miR}-10 \mathrm{a} / \mathrm{b}$ in these cell lines could not be attributable to absence of expression in untreated cells given that the $\mathrm{C}_{\mathrm{t}}$ values for biological replicates were in the range of 30-31 $\left(C_{t}\right.$ values in the range of $35-40$ would be indicative of no expression).

Ectopic overexpression of miR-10a and -10b in neuroblastoma SK-N-BE cells recapitulates ATRA-induced differentiation. Mature miR-10a or $-10 b$ mimics were transfected into SK-N-BE, LAN-5 and SHSY-5Y cells, both individually and in combination, resulting in ectopic overexpression. Over expression of both miRNAs resulted in pronounced increased neurite outgrowth by day 5 post transfection ( $P$-values $<0.001$ or better) (Figure 1$)$. Functional studies using SK-N-BE cells transfected with miR10a, $10 \mathrm{~b}$ or $10 \mathrm{a} / \mathrm{b}$ displayed significant reduction in the rate of cell growth (ascertained by cell counts and acid phosphatase (Figure 2a; Supplementary Figure 3) and colony-forming ability in soft agar (Figure 2b). Notably, expression of two markers of differentiation, GAP43 and TUBB3, increased following miR-10a or $-10 \mathrm{~b}$ transfections, consistent with what transpires following ATRA treatment (Figures $2 \mathrm{c}$ and $\mathrm{d}$ ). In addition, there was a highly significant reduction in MYCN mRNA and protein levels (Figures 2e and f) following ectopic overexpression of the miRNAs, similar to what occurred following ATRA treatment. This is most likely an indirect effect given that the MYCN $3^{\prime}$-UTR lacks target sites for either of these miRNAs. Anti-sense knockdown of $\mathrm{miR}-10 \mathrm{a} / \mathrm{b} 24 \mathrm{~h}$ before ATRA treatment led to a highly significant, but incomplete, reduction in the outgrowth of neurites (Supplementary Figure 4). It is not surprising that $\mathrm{miR}-10 \mathrm{a} / \mathrm{b}$ knockdown did not completely reverse the effects of ATRA given that ectopic overexpression of other ATRAinduced miRNAs, such as $125 \mathrm{~b}$, have been shown to result in neurite outgrowth ${ }^{14}$, Supplementary Figure $2 a$. We conclude, nevertheless, that ectopic overexpression of miR-10a and $-10 b$, both individually and in combination, recapitulates many features of the ATRA-induced differentiation phenotype of SK-N-BE cells.

mRNA expression profiling and gene ontology analysis following miR-10 transfection of SK-N-BE cells. In order to experimentally identify direct targets downregulated by miR-10a, mRNA expression profiling was performed using microarrays representing 24000 protein-coding genes. SK-N-BE cells at either 1 or 7 days post transfection with 

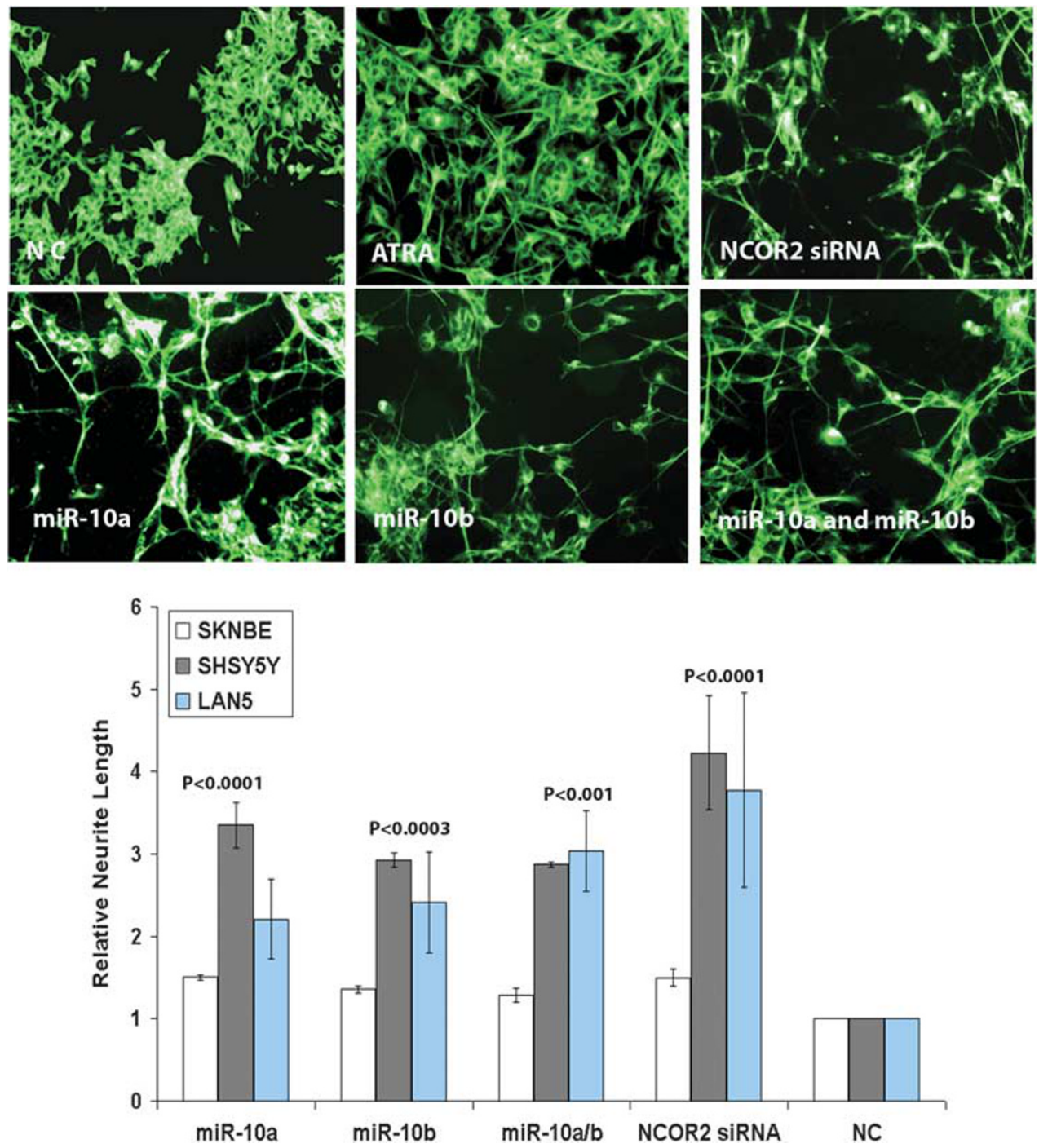

Figure 1 Outgrowth of neurites in neuroblastoma cells. The upper panel shows morphology of SK-N-BE cells following transfections with a negative control (NC) oligonucleotide, treatment with ATRA, and transfections with either NCOR2 siRNA, miR-10a, miR-10b or miR-10a/10b combined. The lower panel displays a bar graph of neurite length measurements following ectopic overexpression of miR-10a, miR-10b, miR-10a + 10b and siRNA-mediated knockdown of NCOR2 in SK-N-BE, SHSY-5Y and LAN5 cell lines at day 5 post transfection. All measurements are relative to the NC, a scrambled oligonucleotide (set as 1.0). All $P$-values are relative to NC and represent the minimum value for the three sets of cell lines

miR-10a were analyzed. There was a statistically significant enrichment for miR-10a-predicted target sites in the set of mRNA sequences, which decreased by twofold or greater following miR-10a transfection at 24 hours $(P=0.03)$ and 7 days $(P=0.00001)$ (Table 1$)$. Interestingly, there were more mRNAs downregulated with predicted targets at day 7 than at day 1 post transfection, indicating that specific mRNAs might be targeted at differential rates. However, the majority $(91.7 \%)$ of mRNAs possessing miR-10a-binding sites that were downregulated after $24 \mathrm{~h}$ remained downregulated at day 7. There was also large numbers of mRNAs downregulated at both $24 \mathrm{~h}(n=845)$ and 7 days post transfection $(n=1629)$ that lacked miR-10a target sites, indicating that ectopic upregulation of this miRNA results in substantial secondary effects. For upregulated mRNAs, there were significantly greater numbers at day 7 than at day 1 post transfection, which also would be because of secondary events. At the 7-day time point, the upregulated mRNAs were significantly inversely correlated with miR-10-binding sites, a correlation which was not statistically significant at day 1 .

Gene ontology analysis on the most significantly upregulated mRNAs (top 20\%) following transfection of SK-N-BE cells with miR-10a indicated that the top enriched term was cell surface signal transduction following correction for multiple comparisons (Supplementary Table 1). For the most significantly downregulated genes (top $20 \%$; both direct and indirect effects), the most significant enrichment term was regulation of transcription (Supplementary Table 1), which explains why ectopic overexpression of miR-10a launches a cascade of secondary alterations to the transcriptome. The decrease in MYCN following miR-10a/b transfection or ATRA 

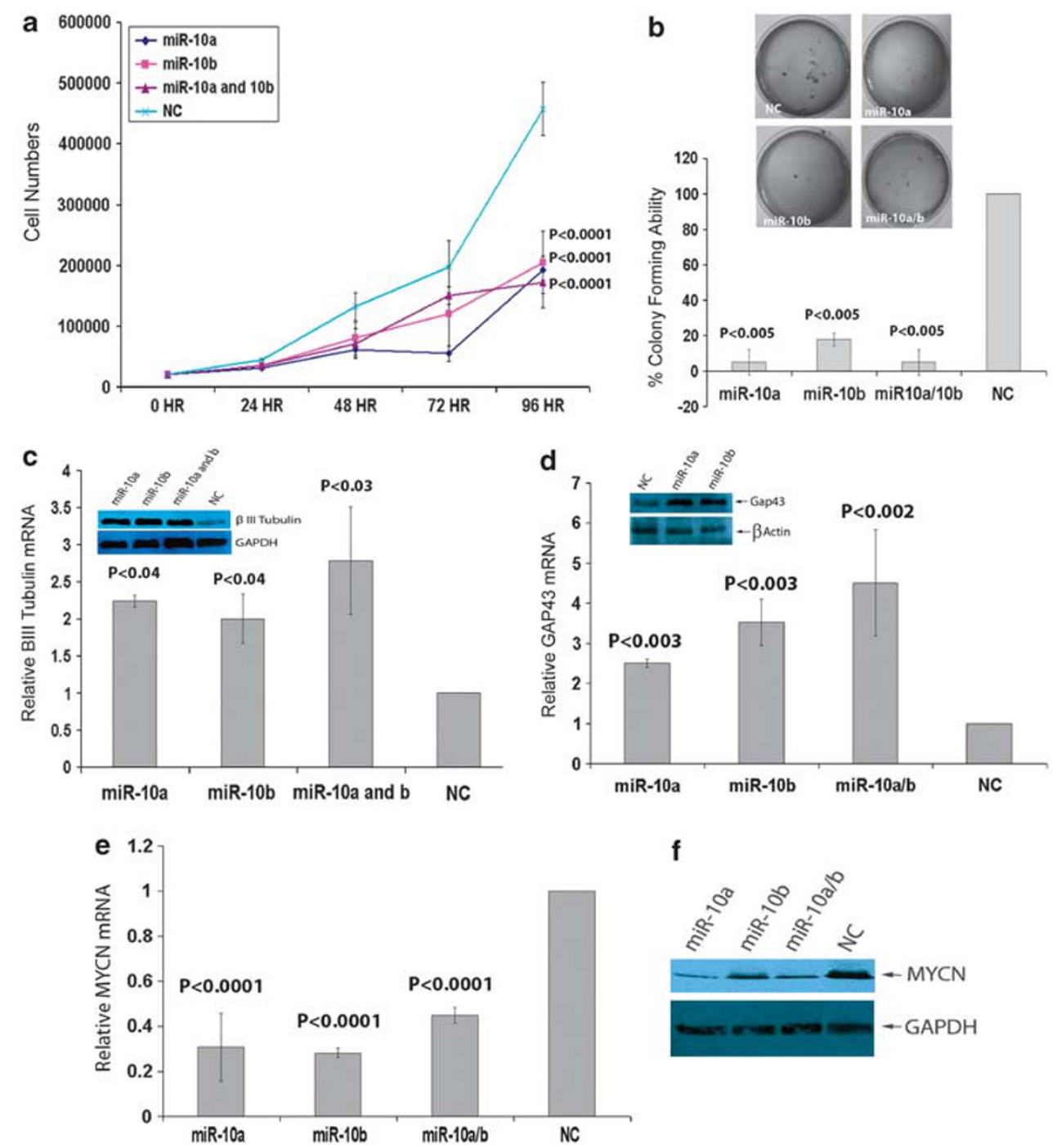

f

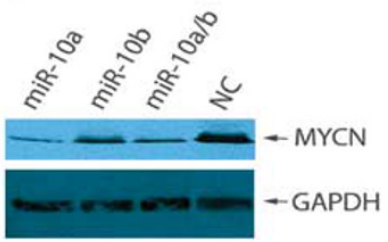

Figure 2 Biological effects of miR-10a and miR-10b ectopic overexpression in SK-N-BE. (a) Growth curve based on cell counts at different time points following transfection of SK-N-BE cells with a negative control (NC) oligonucleotide, miR-10a, miR-10b or combined miR-10a/b mature mimics. Similar results were obtained by cell counting (Supplementary Figure 3). (b) Reduction in colony forming ability in soft agar following transfections. Increase in $\beta$-3-tubulin (TUBB3) (c) and GAP43 (d) mRNA and protein following transfections. (e) Reduction in MYCN mRNA and protein (f) following transfections

Table 1 Effect of 10a transfection on gene expression

\begin{tabular}{|c|c|c|c|}
\hline & Day 1 & Day 7 & No. overlap day 1 and day 7 \\
\hline Total downregulated mRNAs (> twofold) & 977 & 1915 & $811(83.0 \%)$ \\
\hline Observed 10 a targets & 132 & 286 & $121(91.7 \%)$ \\
\hline Expected 10a targets ${ }^{a}$ & 113.7 & 223.0 & 94.3 \\
\hline Significance of overrepresentation $(\mathrm{P})$ & 0.03 & 0.00001 & 0.002 \\
\hline Total upregulated mRNAs ( $>$ twofold) & 1101 & 1663 & $900(81.7 \%)$ \\
\hline Observed $10 \mathrm{a}$ targets & 116 & 148 & $89(76.7 \%)$ \\
\hline Expected 10a targets ${ }^{a}$ & 128.2 & 193.5 & 104.7 \\
\hline Significance of under-representation $(P)$ & 0.14 & 0.0001 & 0.0534 \\
\hline
\end{tabular}

${ }^{a}$ Expected number of binding sites generated by simulation studies based on 1000000 iterations of similar numbers of randomly selected mRNAs and miRNAs

treatment would also impact mRNA and miRNAs levels. In this regard, some MYCN responsive miRNAs, including miR-184, 152 and 190 are upregulated in response to ATRA and would result in further tertiary effects.
One of the downregulated miR-10a/b targets, NCOR2, is a corepressor of transcription, whose downregulation could account for the upregulation of many genes detected by the microarray analyses. From the standpoint of neurite 
outgrowth, NCOR2 (SMRT), is of immediate interest given that neural stem cells derived from NCOR2 homozygous knockout mice exhibit extensive neurite outgrowth and reduction in cell proliferation following 5 days in culture. ${ }^{24}$ For these reasons, NCOR2 was selected for further functional studies.

The miR-10a/10b predicted target site in the $3^{\prime}-$ UTR of NCOR2 is broadly conserved and contains an eight-nucleotide match in the seed region of $m i R-10 a / b$. The downregulation of NCOR2 mRNA following miR-10 overexpression in SK-N-BE was validated by qPCR and at protein level by immunoblotting (Figures $3 a$ and $b$ ). Moreover, a luciferase reporter construct containing a 484 bp region of the NCOR2 3'-UTR (designated Psi/miR-10) showed a significant decrease in luciferase activity when cotransfected with mature miR-10a, $-10 \mathrm{~b}$ or $10 \mathrm{a} / 10 \mathrm{~b}$ mimics in SK-N-BE cells (Figure $3 c$ ). The decrease in luciferase activity was completely abrogated by a $3 \mathrm{bp}$ mutation in the seed region (designated Psi/miR-10mut), leading us to conclude that NCOR2 is a direct target of both miR-10a and $-10 \mathrm{~b}$.

Effects of NCOR2 on neurite outgrowth and cell growth. As already discussed, miR-10a and $-10 \mathrm{~b}$ have many direct and indirect effects on the mRNA transcriptome. In order to assess how downregulation of NCOR2 alone might contribute to neuroblastoma cell differentiation, we performed siRNA-mediated knockdown of NCOR2 in SK-N-BE cells. Transfection of two independent NCOR2 siRNAs into SK-N-BE cells resulted in approximately $60 \%$ reduction in NCOR2 mRNA levels and a significant reduction in protein (Figure 4a). This level of NCOR2 knockdown resulted in a highly significant increase in neurite outgrowth $(P<0.0001)$ (Figure 1$)$ and a reduction in cell growth (Figure 4b). Similar to ATRA-treated cells, siRNA-mediated NCOR2 knockdown resulted in reduced MYCN mRNA and protein (Figure 4c) and increased amounts of the neuronal markers GAP43 and TUBB3 (Figure 4d). Most interestingly, inhibition of NCOR2 resulted in a 14-fold increase in miR-10a $(P<0.0001)$ (Figure $4 \mathrm{~d})$, providing further evidence for the involvement of NCOR2 in the suppression of this miRNA (see proposed mechanism of action model Figure 5).

In order to further validate that NCOR2 is the only target of miR-10a that is required for yielding a differentiated phenotype, we cotransfected an NCOR2 expression construct lacking the $3^{\prime}$-UTR region with $\mathrm{miR}-10 \mathrm{a}$ mature mimics. Transfection of the NCOR2 construct into SK-N-BE cells resulted in increased NCOR2 mRNA and protein, reflecting both endogenous and ectopic molecules (Figure 6a). Cotransfection of NCOR2 plasmid and miR-10a led to an approximate twofold increase in NCOR2 protein over the negative control at $72 \mathrm{~h}$, reflecting only ectopically derived protein (Figure 6a). Cotransfection completely blocked the a

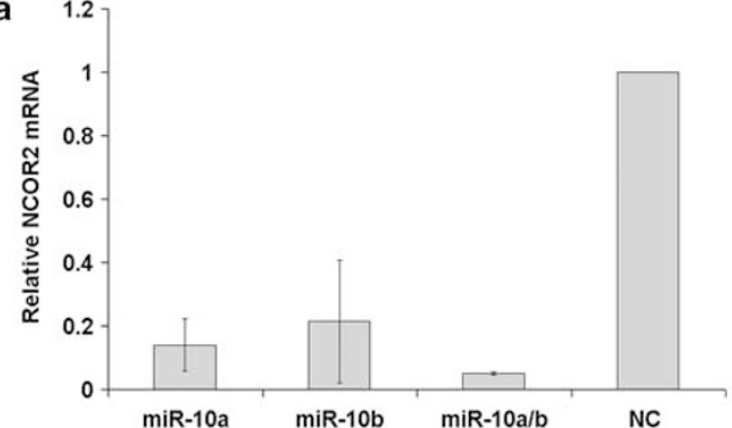

C

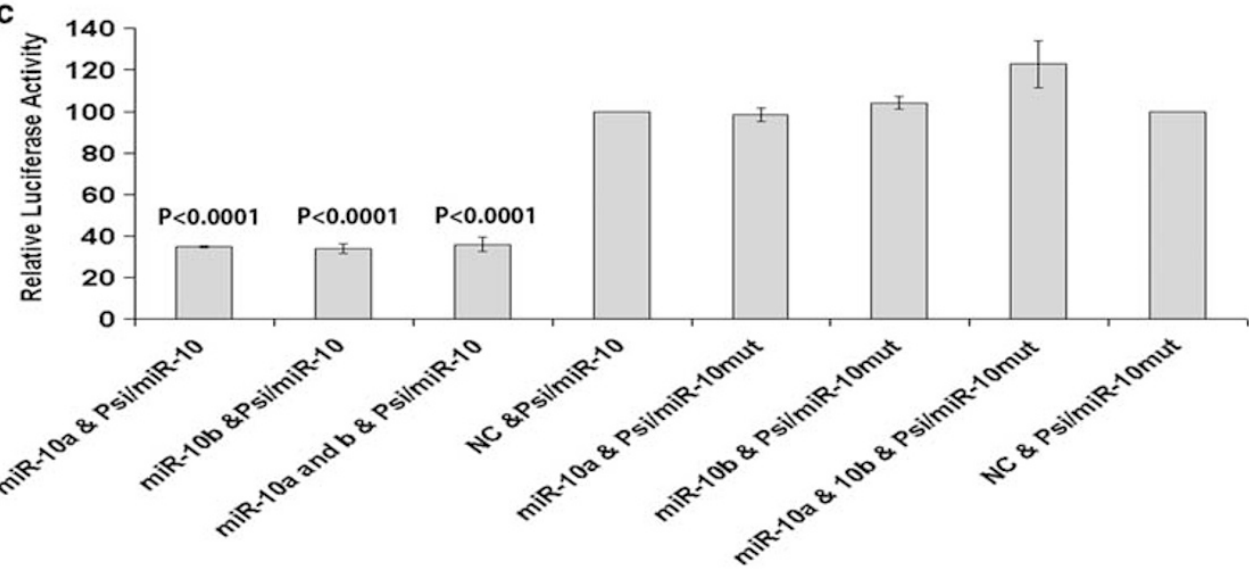

b

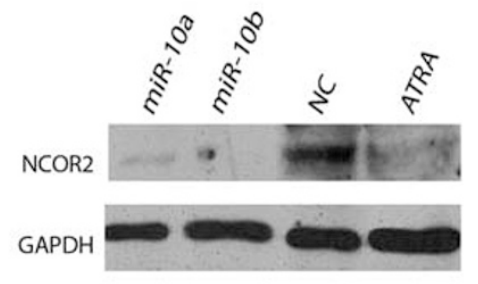



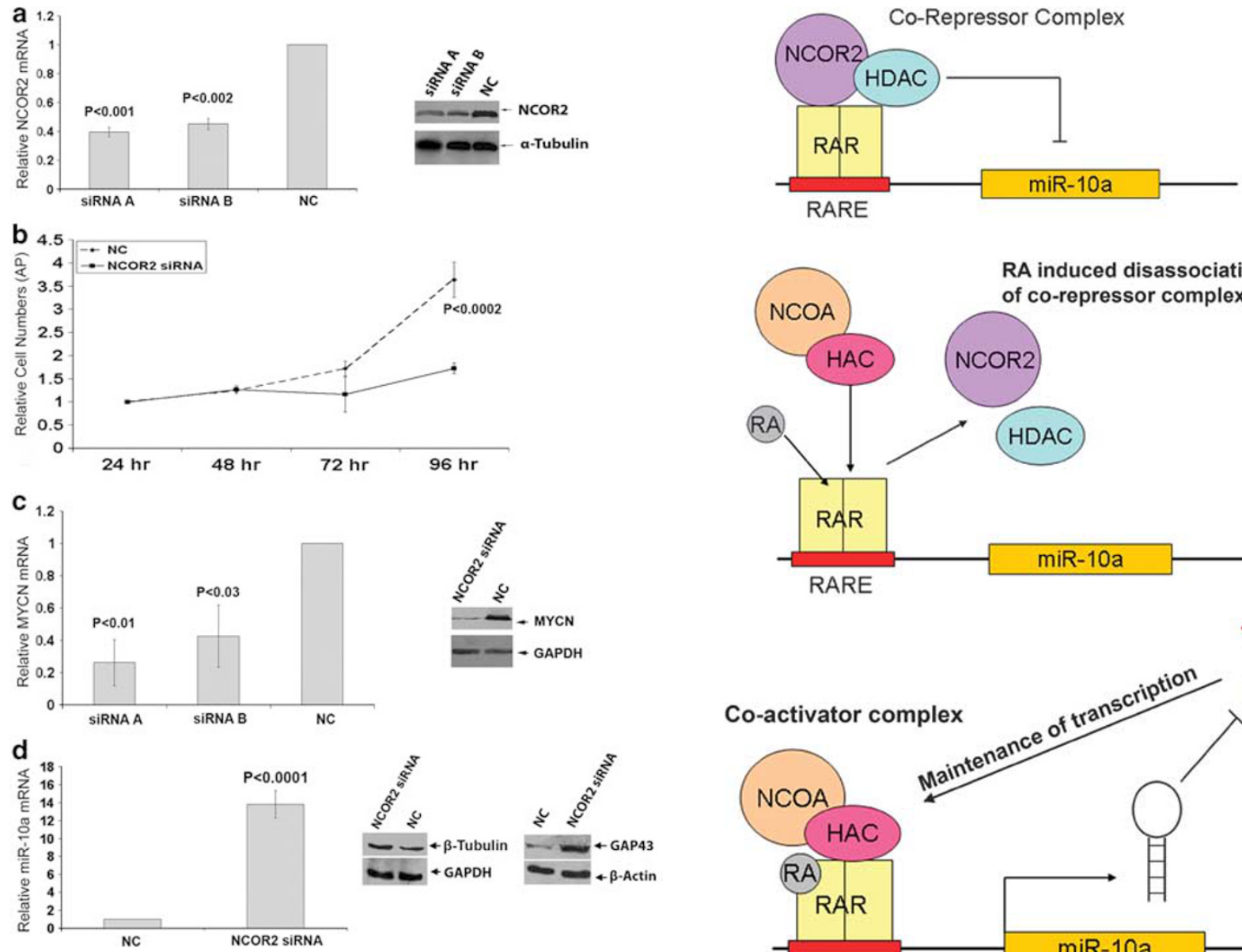

Figure 4 Effects of siRNA-mediated inhibition of NCOR2. (a) Knockdown of NCOR2 mRNA and protein using two different siRNAs. (b) Reduction in cell numbers following NCOR2 knockdown as assessed by an acid phosphatase assay. (c) Reduction in MYCN mRNA and protein after NCOR2 inhibition and (d) increases in miR-10a, TUBB3 and GAP43 expression following siRNA-mediated NCOR2 knockdown

effects of miR-10a, including overexpression of GAP43, TUBB3B and downregulation of MYCN (Figure 6b). Furthermore, at day 5 following cotransfection there was no detectable increase in neurite outgrowth (Figure 6c) or decrease in the rate of accumulation of cell numbers (Figures $6 d$ and e).

MiR-10a and $-10 b$ expression in primary neuroblastoma tumors and association with clinical features. Given that patient survival is related to the degree of differentiation of neuroblastoma tumors, we examined the expression patterns of miR10a/b in a cohort of 145 primary neuroblastoma tumors. As illustrated in Supplementary Figure 5a, miR-10a was significantly under-expressed (1.4-1.9 fold; $P<0.0001)$ in two genetic subtypes that are associated with poor clinical outcome, those with 11q deletion $(n=30$; stage 4) or MYCN amplification ( $n=45$; stage 4), relative to tumors lacking these abnormalities. MiR-10b displayed an inverse pattern of expression in the tumor subgroups from miR-10a (Supplementary Figure 5b). Interestingly, miR-10a maps within a chromosomal region on chromosome $17 q$ that is gained in $\sim 80-90 \%$ of tumors from patients with stage
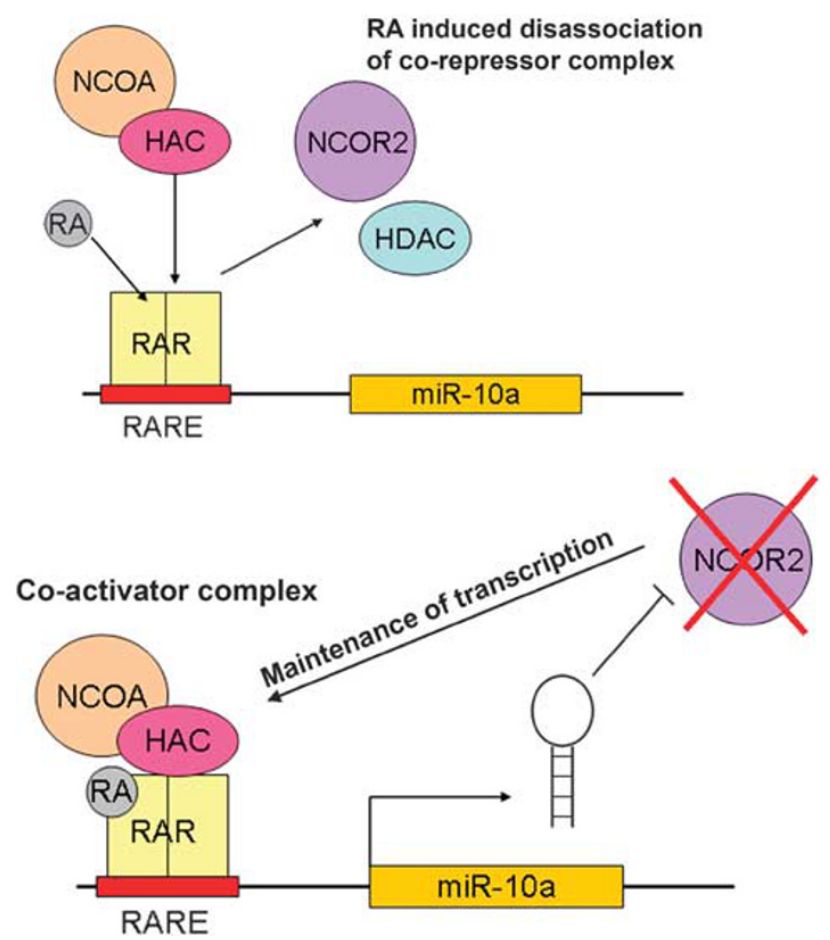

Figure 5 Model for the activation of miR-10a by retinoic acid (RA) and subsequent maintenance of transcriptional activity through direct targeting of NCOR2 $3^{\prime}$-UTR. The upper display shows binding of a corepressor complex consisting of a retinoic acid receptor (RAR), NCOR2, and histone deacetyltransferases (HDAC) to a retinoic acid response element (RARE) upstream to miR-10a. In the middle display, RA causes dissociation and replacement of the corepressor complex with a coactivator complex that activates miR-10a transcription. MiR-10a transcription is maintained through targeting the NCOR2 $3^{\prime}$-UTR (lower display)

four disease, but is nevertheless expressed at significantly $(P<0.0001)$ lower levels in tumors with $17 q$ gain in spite of increased dosage (Supplementary Figure $5 \mathrm{c}$ ). This result indicates that although there might be a gene or set of genes on chromosome $17 q$ that are selectively upregulated by 17q gain, miR-10a expression is downregulated by some unknown mechanism.

In the overall cohort of 145 patients, lower miR-10a expression in tumors was not significantly associated with worse overall (OS) or event-free survival (EFS). However, within the subset of 11q- tumors, lower than median miR-10a expression was associated with poor OS $(P=0.045)$ and displayed a trend towards poor EFS $(P=0.057)$ (Supplementary Figures $5 \mathrm{~d}$ and e). The 11q- tumors represent a distinct genetic subtype of neuroblastoma, which can be subdivided into clinically favorable and unfavorable groups on the basis of mRNA/miRNA expression patterns. ${ }^{25,26}$ No association 

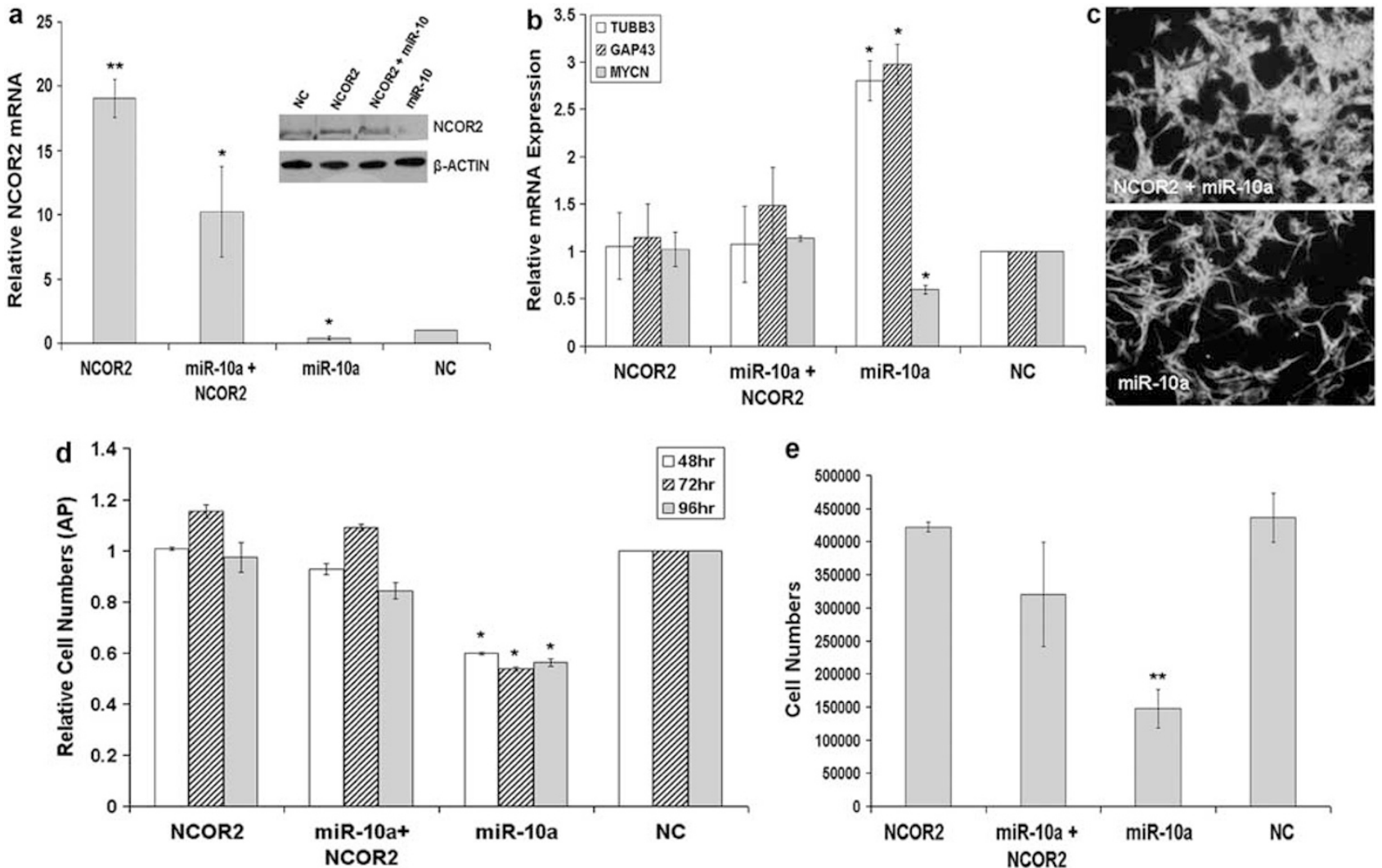

Figure 6 Ectopic overexpression of NCOR2 lacking a 3'-UTR can counteract the effects of miR-10a overexpression. (a) Transfection of the NCOR2 plasmid into SK-N-BE by itself (ectopic + endogenous expression) yielded a higher level of NCOR2 mRNA and protein than cotransfection of the plasmid with miR-10a (ectopic expression only). (b) The NCOR2 plasmid when cotransfected with miR-10a inhibited the overexpression of neuronal markers TUBB3 and GAP43 (as observed when only miR-10a is transfected) and prevented the downregulation of MYCN. (c) Cotransfection of NCOR2 with miR-10a prevented neurite outgrowth. (d) Cotransfection of NCOR2 with miR-10a counteracts the effects of miR-10a on cell viability, as demonstrated by an acid phosphatase assay (d) and confirmed by cell counts taken at 72 hrs (e). Note that approximately 20 -fold ectopic upregulation of NCOR2 by itself has no impact on cell growth. In the above figures NC is a negative control transfected with a scrambled oligonucleotide sequence. ${ }^{*} P<0.05,{ }^{\star \star} P<0.005$ relative to the appropriate $\mathrm{NC}$, absence of asterisk $\left({ }^{*}\right)$ denotes statistical insignificance relative to NC

between miR-10b expression levels and patient survival was discernable. We conclude that although the in vitro effects of $\mathrm{miR}-10 \mathrm{a}$ and $-10 \mathrm{~b}$ are indistinguishable, they have remarkably different patterns of expression in tumor subtypes, with under-expression of miR-10a having a greater influence on patient survival for the 11q- tumor subtype.

\section{Discussion}

Previous studies of miRNA expression profiling of ATRAtreated neuroblastoma cell lines differ in the use of cell lines (SK-N-BE versus SHSY-5Y), analysis platforms (qPCR, microarray or northern blot), and the numbers of miRNAs that were profiled (ranging from 32 to 364 ), thus some variation in the miRNAs determined to be differentially regulated in response to ATRA would not be surpris-

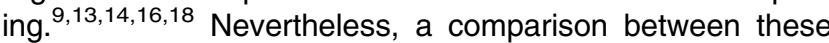
studies revealed that 20 out of 56 miRNAs determined to be up- or downregulated in the current study were also similarly differentially regulated in at least one other study, including miR-10a (Supplementary Table 2). This is not to imply that the miRNAs identified as ATRA responsive in a single study are not valid, only that they have yet to be confirmed.
This study has determined that endogenous overexpression of $\mathrm{miR} 10 \mathrm{a} / \mathrm{b}$ in response to ATRA has a major role in the process of neuroblastoma cell differentiation through the specific targeting and inhibition of NCOR2. MiR-10a and -10b differ by a single nucleotide, which occurs outside of the 'seed' region, thus, it is not surprising that the in vitro biological effects of these miRNAs are indistinguishable following ectopic upregulation in SK-N-BE cells. Both miRNAs lead to phenotypic changes similar to ATRA-induced differentiation, including a significant increase in neurite outgrowth, upregulation of the neuronal differentiation markers GAP43 and $T U B B 3$, the downregulation of $M Y C N$ and a reduction of cell growth. In addition, miR-10a and $-10 \mathrm{~b}$ overexpression launches a cascade of mRNA transcript changes through a complex series of primary and secondary mechanisms. In spite of the vast re-programming of the SK-N-BE cell transcriptome following miR-10a/10b transfections, it is remarkable that siRNA-mediated inhibition of one single direct target, NCOR2, can recapitulate the differentiating phenotypic effects of these miRNAs.

NCOR2 has the ability to bind to hormone receptors, including retinoic acid receptors, recruit corepressor proteins and silence primary response genes. ${ }^{27,28}$ NCOR2 functions 
as a transcriptional corepressor through recruitment of a complex of proteins to DNA promoter regions, including SIN3A/B, and histone deacetylases HDAC1, HDAC2 and HDAC3. ${ }^{29}$ Therapeutic levels of ATRA causes a disruption and displacement of the corepressor complex with a coactivator complex at retinoic acid response elements and subsequent transcriptional activation of adjacent genes. It has been established that miR-10a, which is located on chromosome $17 q$ within the $H O X B 3$ gene, is a retinoic acid receptor target, as knockdown of a retinoic acid receptor in a pancreatic cancer cell line resulted in complete inhibition of miR-10a

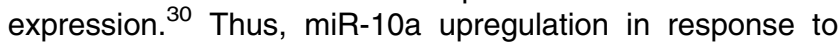
ATRA is a direct consequence of ATRA causing displacement of the corepressor complex with a coactivator complex, leading to chromatin acetylation and concurrent miR-10a transcription (Figure 5). miR-10a can then posttranscriptionally downregulate NCOR2, maintaining the disruption of the corepressor complex in the absence of ATRA. siRNA or miR-10a/b-mediated inhibition of NCOR2 therefore results in the same differentiated phenotype that is caused by ATRA treatment, along with a vast reprogramming of the cellular transcriptome. The involvement of NCOR2 in a corepressor complex that downregulates miR-10a expression indicates that a regulatory feedback loop is in place.

MiR-125b has also recently been implicated in promoting neuronal differentiation of neuroblastoma SH-SY5Y cells through directly repressing multiple key target genes. ${ }^{14}$ There appears to be some level of redundancy in function of miR-125b with miR-10a/b as three of these key target genes (ITCH, GAB2 and MKNK2) have miR-10 seed sites in their $3^{\prime}$-UTRs and are downregulated following ectopic upregulation of $\mathrm{miR}-10 \mathrm{a} / \mathrm{b}$ in our studies. Although not noted in the study by Le et al., ${ }^{14}$ it is highly significant that miR-125b also has a 7-mer conserved seed match in the $3^{\prime}$-UTR of NCOR2 (TargetScan), further explaining why both miRNAs cause neural cell differentiation.

Analysis of the expression of miR-10a and -10b in primary neuroblastoma tumors, along with patient survival, indicates that these miRNAs differ in both their patterns of expression in genetic subtypes and in their associations with clinical outcome. MiR-10b expression is not correlated with patient survival, whereas low expression of miR-10a shows a trend towards lower OS and EFS for patients with 11q- tumors. Although miR-10a maps to a region on chromosome $17 q$ that commonly undergoes increased copy-number in most high-risk neuroblastomas, miR-10a expression is significantly lower in tumors with $17 q$ gain relative to tumors with diploid $17 q$ copynumber status, indicating that a mechanism exists to counteract the gain in dosage. Thus, it appears to be vital for unfavorable tumors, particularly of the 11q- subtype, to have low miR-10a expression levels, presumably because of the positive effects on differentiation. In contrast, miR-10b is expressed at higher levels in less favorable neuroblastoma tumors and is not associated with lower patient survival even though this miRNA causes in vitro biological effects that are indistinguishable from miR-10a. It is tempting to speculate that the single nucleotide difference between miR-10a and $-10 \mathrm{~b}$, which occurs outside of the seed region has led to different secondary effects of these miRNAs that might account for the differences in their distribution in tumor subtypes.
It is also of interest that the role of $\mathrm{miR}-10 \mathrm{a} / \mathrm{b}$ in cancer appears to be tumor and cell context dependent, as miR-10a has been reported to be under-expressed in hematological malignancies such as $\mathrm{CML},{ }^{31}$ whereas both miRNAs have been reported to be overexpressed in a variety of solid tumors. ${ }^{32}$ Overexpression of miR-10b has been associated with breast cancer metastasis, ${ }^{33}$ although this view has been challenged. ${ }^{34}$ In this regard it is interesting that miR-10b is marginally overexpressed in tumors with either MNA or $11 \mathrm{q}$ $\mathrm{LOH}$ from patients with metastatic disease, relative to locoregional tumors lacking these unfavorable genetic alterations.

In conclusion, we have identified two miRNAs, which induce differentiation and inhibit cell proliferation in neuroblastoma cell lines, which are capable of undergoing ATRA-induced differentiation, and validate NCOR2 as a direct target of miR10a and -10b. Interestingly, NCOR2 downregulation leads to the indirect suppression of MYCN, a potent oncoprotein in neuroblastoma. MiR-10a/b could be of potential therapeutic benefit if targeted delivery of these miRNAs is achievable, given their pro-differentiation and anti-proliferative effects.

\section{Materials and Methods}

ATRA treatment. SK-N-BE cells were treated with $5 \mu \mathrm{mol}$ of ATRA. ATRA (Sigma, St. Louis, MO, USA) was continuously administered by replacing the medium every $24 \mathrm{~h}$ for 7 days. Cell morphology was monitored using the neuronal specific antibody $\beta$ III Tubulin (Abcam, Cambridge, MA, USA), followed by staining with an anti-rabbit Alexa 488-conjugated antibody (Invitrogen, Carlsbad, CA, USA).

microRNA and siRNA transfections. Reverse transfections of microRNAs and siRNAs were carried out at final concentrations of $30 \mathrm{nM}$ for miRNAs and $10 \mathrm{nM}$ for siRNAs in optiMEM (Gibco, Carlsbad, CA, USA). Separate NeoFX and optiMEM solutions were prepared and then mixed together at equal volumes. A volume of 200 or $20 \mu \mathrm{l}$ of this solution was then added to a well from either a 6- or 96-well plate, respectively, which was then over-layed with neuroblastoma cell suspension and incubated at $37^{\circ} \mathrm{C}$. A flourescein-labeled double stranded oligonucleotide (Invitrogen) was transfected into cell lines under the conditions stated above in order to determine the transfection efficiencies. Approximately $78 \%$ of cells exhibited luciferase activity $48 \mathrm{~h}$ post transfection.

RNA extractions. Total RNA with conservation of small RNAs was extracted using the miRNeasy kit (Qiagen, West Sussex, UK) as per manufacturer's instructions. RNA quality and quantity was measured using the Nanodrop Spectrophotometer (Thermoscientific, Loughborough, UK) and RNA dilutions were made up in nuclease-free water.

Stem-loop reverse transcription and real-time PCR. Reverse transcription of $50 \mathrm{ng}$ of RNA from ATRA-treated SK-N-BE cells at Day 0, Day 3 and Day 7 was carried out using the stem-loop Multiplex primer pools (Applied Biosystems), allowing reverse transcription of 48 different miRNA in each of eight RT pools. Reverse transcription for individual qPCRs was carried out using $50 \mathrm{ng}$ of total RNA and the High-Capacity Reverse Transcription Kit (Applied Biosystems), RT specific primers for microRNAs miR-10a and -10b (Applied Biosystems) were used for all microRNA reverse transcription. The microRNA qPCR screen was carried out on the 7900HT Fast Realtime System using TaqMan Low-Density Arrays (TLDA) (TaqMan TLDA MicroRNA Assays AIF v5 containing 364 human microRNAs assays; Applied Biosystems).

Individual qPCRs were carried out on the $7900 \mathrm{HT}$ Fast Realtime System (Applied Biosystems) using GAP43-, $\beta$ III Tubulin-, MYCN- and NCOR2-specific Taqman gene expression assays (Applied Biosystems) and miR-10a- and -10bspecific Taqman microRNA assays (Applied Biosystems). RPLPO and RNU66 were used for normalization in gene and microRNA expression studies, respectively. A relative fold change in expression of the target gene transcript was determined using the comparative cycle threshold method $\left(2^{-\Delta \Delta C T}\right)$. 
Western blot. Total protein was isolated from cells using a radioimmunoprecipitation assay (RIPA) lysis buffer (Sigma). Cell pellets were washed with PBS and solubilized in RIPA for 30 min. Protein concentration was measured using the BCA assay from Pierce. Proteins were fractionized on 6 or $10 \%$ polyacrylamide gels, and blotted onto nitrocellulose membrane. The membrane was probed with the Anti-NCOR2 Antibody (Abcam), Anti-GAP43 (Abcam), Anti-MYCN (Santa Cruz, Santa Cruz, CA, USA), Anti- $\beta$ III Tubulin and anti-GAPDH (Abcam), anti- $\alpha$-Tubulin, and anti-Actin (used for loading controls). Signal was detected using immoblion western (Millipore, Billerica, MA, USA).

Gene expression analysis. The SuperScript Double-Stranded cDNA Synthesis Kit (Invitrogen, 11917-020) was used to synthesize cDNA from $10 \mu \mathrm{g}$ total RNA according to the manufacturer's instructions. Cy3 labeling of cDNA was carried out using the NimbleGen One-Colour DNA Labelling Kit (NimbleGen, Madison, WI, USA). A measure of $4 \mu \mathrm{g}$ of labeled cDNA was hybridized to the Homo Sapiens $4 \times 72 \mathrm{~K}$ gene expression array (Roche-NimbleGen, Madison, WI, USA) for $16 \mathrm{~h}$. Following washing, arrays were scanned using the GenePix 4000B scanner, and expression values were obtained using the Robust Multi-Chip Average algorithm ${ }^{35}$ in NimbleScan 2.4

Growth curve. For cell numbers, cells were set up in 6-well plates. Cells were seeded at equal densities. When carrying out transfections using the microRNA mimics, each time point was set up with a nontransfected (with transfection reagent) and a scrambled oligonucleotide control (negative 1). Cells were trypsinised from 6 -well plates at 24, 48, 72 and $96 \mathrm{~h}$ time points. Cells were resuspended in $1 \mathrm{ml}$ of media. The Scepter cell counter (Millipore) was used to count cell numbers. Counts from triplicate wells were averaged.

Acid phosphatase assay. Cells were transfected in 96-well plates (103 cells per well). At $96 \mathrm{~h}$ wells were washed twice with PBS and $10 \mathrm{mM}$-nitrophenol phosphate in $0.1 \mathrm{M}$ sodium acetate with $0.1 \%$ triton $\mathrm{X}-100$ was added. Plates were incubated at $37^{\circ} \mathrm{C}$ for $2 \mathrm{~h}$ and the reaction was stopped with $50 \mu \mathrm{l} 1 \mathrm{M}$ sodium hydroxide per well. Absorbance was measured at $405 \mathrm{~nm}$. Values were normalized to the scrambled oligonucleotide negative control.

Colony forming efficiency. A $1 \%$ agarose solution was plated on $10 \mathrm{~cm}^{2}$ petri dish until the plate was completely covered. Cells were plated at $1.5 \times 10^{6}$ in $10 \mathrm{ml}$ of culture media, and media was replaced every $4-5$ days, for 21 days. Colonies were stained with $0.1 \%$ crystal violet. Plates were photographed; colonies were counted in replicate studies and counts normalized to the negative scrambled control.

Immunostaining. Cells were fixed in $4 \%$ paraformaldhyde 5 days after transfection. Cells were permeabilised using Trition X-100 (Sigma), then blocked for $30 \mathrm{~min}$ in BSA. Samples were incubated in the primary $\beta$-III Tubulin rabbit antibody (Abcam) overnight at $4^{\circ} \mathrm{C}$, followed by incubated in the secondary Alexa Fluor 488 AntiRabbit fluorescent antibody (Invitrogen) for $1 \mathrm{~h}$ at room temperature. To stain nuclei the cells were incubated for 5 min with DAPI (Sigma) and then washed in PBS.

Neuronal outgrowth assay. Cells were imaged using Cellomics ArrayScan VTi instrument (Thermofisher, Loughborough, UK) equipped with $10 \times$ PlanApo objective lens (NA 0.45), 120W Hg arc illumination source (EXFO) and a monochrome CCD camera (Hamamatsu Orca AG, Welwyn Garden City, UK). Images of 49 fields of view were registered from each well with $1024 \times 1024$ pixel resolution ( $645 \mathrm{~nm}$ pixel size). The fluorescence of DAPI (nuclei) was excited using $375-400 \mathrm{~nm}$ light and collected in $425-460$ band, whereas the fluorescence of Alexa 488 (whole cells) was excited using 470-490 nm light and collected in 500550 band. The images of nuclear fluorescence were preprocessed using rank leveling ( 25 pixel window) for background subtraction. The nuclei, cell bodies and neurites were segmented using fixed intensity thresholds. The nuclei were used as seeds for morphological separation of adjacent cell bodies. The neurites were then delineated with skeletonization of their binary masks. The total neurite length (per cell) was used to estimate neurite outgrowth. The length was normalized to the respective value for the nontreated cells. All image-processing operations were executed using Neuronal Profiling BioApplication of the Discovery Toolbox Software (Thermofisher).

Luciferase reporters. A $484 \mathrm{nt}$ long region of the $3^{\prime}$-UTR of NCOR2 containing the predicted miR-10a/10b binding site was inserted into the dual luciferase PsiCheck2 reporter vector (Promega, Madison, WI, USA), designated
Psi/miR-10 (Supplementary Figure 6a). As a negative control, a 3 nt mutation was introduced into the miR-10a/10b seed region of this sequence, designated Psi/miR10 mut (Supplementary Figure 6b). SK-N-BE cells were plated in 6-well format, and cotransfected using Lipofectamine 2000 with either $2 \mu \mathrm{g}$ of Psi/miR-10 or Psi/ miR-10mut, along with either $30 \mathrm{~nm}$ of either the Pre-miR for $10 \mathrm{a}, 10 \mathrm{~b}$, both Pre-miRs together or a scrambled oligonucleotide negative control. Luciferase activity was measured after $72 \mathrm{~h}$ using a Viktor Plate Reader.

miR-10a/NCOR2 cotransfection. A plasmid containing the coding sequence without the $3^{\prime}$-UTR of NCOR2 was obtained from OriGene (Rockville, MD, USA) and cotransfected with miR-10a or a scrambled control oligonucleotide using Lipofectamine 2000 according to the manufacturer's instructions. Cells were harvested for RNA and protein at $72 \mathrm{~h}$ post transfection. Cell viability was carried out using the cell counting technique and acid phosphatase assays at 24, 48, 72 and $96 \mathrm{~h}$ post transfection.

Patient cohort. Tumor material was obtained from either the Children's Oncology Group Tumor Bank ( $n=108)$, or the Tumor Bank at Our Lady's Children's Hospital, Dublin $(n=37)$. Patients were treated under either the US neuroblastoma treatment protocol or the European treatment protocol between 1998-2004. This work was approved by the Research Ethics Committee of the Royal College of Surgeons on 16th October 2007 (application No. REC241) and by the Research Ethics Committee of Our Lady's Children's Hospital on 5 August 2008 (application number GEN/70/07). The tumor cohort came from patients with stage $4(n=74)$, stage $3(n=31)$ and stages 1,2 and $4 \mathrm{~s}(n=40)$ disease (INSS). Tumor RNA was extracted using the miRNeasy kit (Qiagen) according to the manufacturer's instructions.

Array CGH. DNA copy number variation was carried by array CGH on 145 neuroblastoma tumors as previously described by Buckley et $\mathrm{al}^{25}{ }^{25}$ using a 72000 feature array (NimbleGen) and protocols originally described by Selzer et al. ${ }^{36}$

MicroRNA target prediction. Targetscan V5.1 microRNA target prediction programme was used throughout this study.

\section{Conflict of Interest}

The authors declare no conflict of interest.

Acknowledgements. This work was supported in part by Science Foundation Ireland (07/IN.1/B1776), Children's Medical and Research Foundation, Cancer Research Ireland and the US National Institutes of Health (5R01CA127496). TB was supported by a Marie Curie Cofund EU Fellowship (CEMP; jointly funded by the National Biophotonics and Imaging Platform, Higher Education Authority, Ireland).

1. Baek D, Villen J, Shin C, Camargo FD, Gygi SP, Bartel DP. The impact of microRNAs on protein output. Nature 2008; 455: 64-71.

2. Bartel DP. MicroRNAs: genomics, biogenesis, mechanism, and function. Cell 2004; 116 : 281-297.

3. Lim LP, Lau NC, Garrett-Engele P, Grimson A, Schelter JM, Castle J et al. Microarray analysis shows that some microRNAs downregulate large numbers of target mRNAs. Nature 2005; 433: 769-773.

4. Miska EA, Alvarez-Saavedra E, Townsend M, Yoshii A, Sestan N, Rakic P et al. Microarray analysis of microRNA expression in the developing mammalian brain. Genome Biol 2004; 5: R68.

5. Sempere LF, Freemantle S, Pitha-Rowe I, Moss E, Dmitrovsky E, Ambros V. Expression profiling of mammalian microRNAs uncovers a subset of brain-expressed microRNAs with possible roles in murine and human neuronal differentiation. Genome Biol 2004; 5: R13.

6. Li X, Jin P. Roles of small regulatory RNAs in determining neuronal identity. Nat Rev Neurosci 2010; 11: 329-338.

7. Edsjo A, Holmquist L, Pahlman S. Neuroblastoma as an experimental model for neuronal differentiation and hypoxia-induced tumor cell dedifferentiation. Semin Cancer Biol 2007; 17: 248-256

8. Nagai J, Yazawa T, Okudela K, Kigasawa H, Kitamura H, Osaka H. Retinoic acid induces neuroblastoma cell death by inhibiting proteasomal degradation of retinoic acid receptor alpha. Cancer Res 2004; 64: 7910-7917.

9. Chen Y, Stallings RL. Differential patterns of microRNA expression in neuroblastoma are correlated with prognosis, differentiation, and apoptosis. Cancer Res 2007; 67: 976-983.

10. Thiele CJ, Reynolds CP, Israel MA. Decreased expression of N-myc precedes retinoic acid-induced morphological differentiation of human neuroblastoma. Nature 1985; 313 404-406. 
11. Seeger RC, Brodeur GM, Sather H, Dalton A, Siegel SE, Wong KY et al. Association of multiple copies of the $\mathrm{N}$-myc oncogene with rapid progression of neuroblastomas. $\mathrm{N} E n g / \mathrm{J}$ Med 1985; 313: 1111-1116.

12. Brodeur GM, Seeger RC, Schwab M, Varmus HE, Bishop JM. Amplification of N-myc in untreated human neuroblastomas correlates with advanced disease stage. Science 1984; 224: 1121-1124.

13. Laneve P, Di Marcotullio L, Gioia U, Fiori ME, Ferretti E, Gulino A et al. The interplay between microRNAs and the neurotrophin receptor tropomyosin-related kinase $C$ controls proliferation of human neuroblastoma cells. Proc Natl Acad Sci USA 2007; 104: 7957-7962.

14. Le MT, Xie H, Zhou B, Chia PH, Rizk P, Um M et al. MicroRNA-125b promotes neuronal differentiation in human cells by repressing multiple targets. Mol Cell Biol 2009; 29: 5290-5305

15. Evangelisti C, Florian MC, Massimi I, Dominici C, Giannini G, Galardi S et al. MiR-128 up-regulation inhibits Reelin and DCX expression and reduces neuroblastoma cell motility and invasiveness. FASEB J 2009; 23: 4276-4287.

16. Beveridge NJ, Tooney PA, Carroll AP, Tran N, Cairns MJ. Down-regulation of miR-17 family expression in response to retinoic acid induced neuronal differentiation. Cell Signal 2009; 21: 1837-1845.

17. Loven J, Zinin N, Wahlstrom T, Muller I, Brodin P, Fredlund E et al. MYCN-regulated microRNAs repress estrogen receptor-alpha (ESR1) expression and neuronal differentiation in human neuroblastoma. Proc Natl Acad Sci USA 2010; 107: 1553-1558.

18. Chen H, Shalom-Feuerstein R, Riley J, Zhang SD, Tucci P, Agostini M et al. miR-7 and miR-214 are specifically expressed during neuroblastoma differentiation, cortical development and embryonic stem cells differentiation, and control neurite outgrowth in vitro. Biochem Biophys Res Commun 2010; 394: 921-927.

19. Das S, Foley N, Bryan K, Watters KM, Bray I, Murphy DM et al. MicroRNA mediates DNA demethylation events triggered by retinoic acid during neuroblastoma cell differentiation. Cancer Res 2010; 70: 7874-7881.

20. Shah N, Sukumar S. The Hox genes and their roles in oncogenesis. Nat Rev Cancer 2010; 10: $361-371$.

21. Garzon R, Pichiorri F, Palumbo T, luliano R, Cimmino A, Aqeilan R et al. MicroRNA fingerprints during human megakaryocytopoiesis. Proc Natl Acad Sci USA 2006; 103: 5078-5083.

22. Ma L, Reinhardt F, Pan E, Soutschek J, Bhat B, Marcusson EG et al. Therapeutic silencing of miR-10b inhibits metastasis in a mouse mammary tumor model. Nat Biotechnol 2010; 28: $341-347$.
23. Tsang J, Zhu J, van Oudenaarden A. MicroRNA-mediated feedback and feedforward loops are recurrent network motifs in mammals. Mol Cell 2007; 26: 753-767.

24. Jepsen K, Solum D, Zhou T, McEvilly RJ, Kim HJ, Glass CK et al. SMRT-mediated repression of an $\mathrm{H} 3 \mathrm{~K} 27$ demethylase in progression from neural stem cell to neuron. Nature 2007; 450: 415-419.

25. Buckley PG, Alcock L, Bryan K, Bray I, Schulte JH, Schramm A et al. Chromosomal and miRNA expression patterns reveal biologically distinct subgroups of 11q- neuroblastoma. Clin Cancer Res 2010; 16: 2971-2978.

26. Fischer M, Bauer T, Oberthur A, Hero B, Theissen J, Ehrich M et al. Integrated genomic profiling identifies two distinct molecular subtypes with divergent outcome in neuroblastoma with loss of chromosome 11q. Oncogene 2010; 29: 865-875.

27. Chen JD, Evans RM. A transcriptional co-repressor that interacts with nuclear hormone receptors. Nature 1995; 377: 454-457.

28. Chen JD, Umesono K, Evans RM. SMRT isoforms mediate repression and anti-repression of nuclear receptor heterodimers. Proc Natl Acad Sci USA 1996; 93: 7567-7571.

29. Nagy L, Kao HY, Chakravarti D, Lin RJ, Hassig CA, Ayer DE et al. Nuclear receptor repression mediated by a complex containing SMRT, mSin3A, and histone deacetylase. Cell 1997; 89: 373-380.

30. Weiss FU, Marques IJ, Woltering JM, Vlecken DH, Aghdassi A, Partecke LI et al. Retinoic acid receptor antagonists inhibit miR-10a expression and block metastatic behavior of pancreatic cancer. Gastroenterology 2009; 137: 2136-2145 e2131-2137.

31. Agirre X, Jimenez-Velasco A, San Jose-Eneriz E, Garate L, Bandres E, Cordeu L et al. Down-regulation of hsa-miR-10a in chronic myeloid leukemia CD34+ cells increases USF2-mediated cell growth. Mol Cancer Res 2008; 6: 1830-1840.

32. Lund AH. miR-10 in development and cancer. Cell Death Differ 2010; 17: 209-214.

33. Ma L, Teruya-Feldstein J, Weinberg RA. Tumour invasion and metastasis initiated by microRNA-10b in breast cancer. Nature 2007; 449: 682-688.

34. Gee HE, Camps C, Buffa FM, Colella S, Sheldon H, Gleadle JM et al. MicroRNA-10b and breast cancer metastasis. Nature 2008; 455: E8-E9; author reply E9.

35. Irizarry RA, Hobbs B, Collin F, Beazer-Barclay YD, Antonellis KJ, Scherf $U$ et al. Exploration, normalization, and summaries of high density oligonucleotide array probe level data. Biostatistics 2003; 4: 249-264.

36. Selzer RR, Richmond TA, Pofahl NJ, Green RD, Eis PS, Nair P et al. Analysis of chromosome breakpoints in neuroblastoma at sub-kilobase resolution using fine-tiling oligonucleotide array CGH. Genes Chromosomes Cancer 2005; 44: 305-319.

\section{Supplementary Information accompanies the paper on Cell Death and Differentiation website (http://www.nature.com/cdd)}

